\title{
Spatio-temporal pulses of the Laguerre-Sech shape in a graded- index cubic-quintic medium
}

\author{
Jerzy Jasiński, Małgorzata Kościesza \\ Faculty of Physics, Warsaw University of Technology, Koszykowa 75, 00-662 Warszawa,
}

Received June 19, 2010; accepted September 15, 2010; published September 30, 2010

\begin{abstract}
In the paper the propagation of a $(3+1)$ D pulse in a cubicquintic medium with linear modulation of permittivity is considered. Using a canonical method we obtain a class of exact solutions of EulerLagrange equations corresponding to modes of a linear graded-index waveguide of any order. Assuming small deviations from stationary solutions, we obtain two frequencies of normal oscillations. The properties of these solutions are discussed.
\end{abstract}

In a cubic-quintic [CQ] medium the envelope $U$ of a $(3+1) \mathrm{D}$ pulse propagating along $z$-direction satisfies a generalized nonlinear Schrödinger equation (NSE) [1-5]:

$$
\begin{aligned}
& 2 i \frac{\partial U}{\partial z}+\frac{1}{k}\left(\frac{\partial^{2} U}{\partial x^{2}}+\frac{\partial^{2} U}{\partial y^{2}}\right)-k_{2} \frac{\partial^{2} U}{\partial t^{2}}+ \\
& 2 \varepsilon_{2}\left(|U|^{2}-\frac{|U|^{4}}{I_{s}}\right) U-k \Omega\left(x^{2}+y^{2}\right) U=0 .
\end{aligned}
$$

To reduce problems with the stability of solutions [2-4] let us take $\varepsilon_{2} \cdot k_{2}<0$ and assume an axially symmetric graded-index profile of the linear part of the refractive index [5], so the total permittivity is (where $r^{2}=x^{2}+y^{2}$ ):

$$
\varepsilon(r,|U|)=\varepsilon_{L}\left(1-\Omega^{2} r^{2}+\frac{2 \varepsilon_{2}}{k}\left(|U|^{2}-\frac{|U|^{4}}{I_{s}}\right)\right) .
$$

Nevertheless NSE in the form (1) has no analytical solutions, it is possible to describe the evolution of the considered pulse approximately. To do so we can apply the canonical method [2-7]. For the axially symmetric field $U(r, z, t)=R(r, z, t) \cdot \exp (i \cdot \Phi(r, z, t))$ the Lagrange density function $L$ corresponding to Eq. (1) has the form [5-7]:

$$
\begin{gathered}
L=R^{2} \Phi_{z}-\frac{k_{2}\left(R_{t}^{2}+R^{2} \Phi_{t}^{2}\right)}{2}+\frac{R_{r}^{2}+R^{2} \Phi_{r}^{2}}{2 k}+ \\
\frac{k \Omega^{2} r^{2} R^{2}}{2}-\varepsilon_{2}\left(\frac{R^{4}}{2}-\frac{R^{6}}{3 I_{s}}\right) .
\end{gathered}
$$

(indices $z$ and $t$ denote differentiation over $z$ and $t$ ).

The next step is to postulate the proper trial function. Let us choose the real field amplitude $R$ in the form:

$$
R=b \cdot L_{m}\left(\frac{r^{2}}{a^{2}}\right) \operatorname{Exp}\left(-\frac{r^{2}}{2 a^{2}}\right) \cdot \operatorname{Sech}\left(\frac{t}{w}\right) .
$$

In the above function $b=b(z)=|U(0, z, 0)|$ denotes the height of the pulse, $a=a(z)$ - its spatial and $w=w(z)$ - its temporal width. The Laguerre polynomial of the $m$-th order $L_{m}$ appearing in (4), multiplied by the Gaussian function, composes the solution of the transverse (linear) part of Eq. (1). An analogous solution of the longitudinal (nonlinear) part of the NSE equation for the Kerr case $\left(I_{s}=\infty\right)$ is described by the Sech function. Let us assume the phase of the trial function as:

$$
\Phi=\beta+r^{2} \alpha+t^{2} \theta
$$

where $\beta=\beta(z)$ denotes the propagation term while $\alpha=$ $\alpha(z)$ and $\theta=\theta(z)$ are spatial and temporal curvatures. All field parameters can change during propagation.

Integration of the Lagrange density over transverse and longitudinal coordinates gives the reduced Lagrangian:

$$
\begin{gathered}
\langle L\rangle=2 \pi \int_{0}^{\infty} r d r \int_{-\infty}^{\infty} L(z, r, t) d t, \\
\langle L\rangle=2 \pi a^{2} b^{2} w\left(\beta^{\prime}+h_{a}\left(\frac{1}{2 k a^{2}}+a^{2}\left(\alpha^{\prime}+\frac{2 \alpha^{2}}{k}+\frac{k \Omega^{2}}{2}\right)\right)\right. \\
\left.+\frac{\pi^{2} w^{2}\left(\theta^{\prime}-2 k_{2} \theta^{2}\right)}{2}-\frac{k}{6 w^{2}}-\frac{\varepsilon_{2} h_{p} b^{2}}{6}+\frac{8 \varepsilon_{2} h_{s} b^{4}}{135 I_{s}}\right) .
\end{gathered}
$$

In the last expression prime ' denotes differentiation over $z$ while $h_{a}, h_{s}$ and $h_{p}$ are coefficients obtained from integrals contained in Laguerre polynomials. Using their definition [8] we calculate:

$$
\begin{aligned}
& h_{a}=2 n+1, \\
& h_{p}=1,1 / 2,11 / 32, \ldots \\
& h_{s}=1,29 / 81,467 / 2187, \ldots
\end{aligned} \quad \text { for } n=0,1,2, \ldots,
$$

(unfortunately, for $h_{s}$ and $h_{p}$ we cannot obtain a recursive formula, so we write their value for the lowest orders).

The evolution of any parameter $\xi$ of the trial function is determined by an appropriate Euler-Lagrange equation:

$$
\partial\langle L\rangle / \partial \xi-\partial / \partial z \partial\langle L\rangle / \partial \xi_{z}-\partial / \partial t \partial\langle L\rangle / \partial \xi_{t}=0 \text {. Taking }
$$
as $\xi$, successively $\beta, b, \alpha, a, \theta$ and $w$, we derive six equations which after certain algebra give:

$$
\begin{gathered}
\left(2 \pi a^{2} b^{2} w\right)_{z}=0, \\
\beta^{\prime}=\frac{k_{2}}{3 w^{2}}-\frac{h_{a}}{k a^{2}}+\frac{7 \varepsilon_{2} h_{p} b^{2}}{12}-\frac{16 \varepsilon_{2} h_{s} b^{4}}{45 I_{s}},
\end{gathered}
$$




$$
\begin{gathered}
\alpha=\frac{k a^{\prime}}{2 a}, \\
\alpha^{\prime}+\frac{2 \alpha^{2}}{k}=-\frac{k \Omega^{2}}{2}+\frac{1}{2 k a^{4}}-\frac{h_{p} \varepsilon_{2} b^{2}}{6 h_{a} a^{2}}+\frac{16 h_{s} \varepsilon_{2} b^{4}}{135 h_{a} I_{s} a^{2}}, \\
\theta=-\frac{w^{\prime}}{2 k_{2} w}, \\
\theta^{\prime}-2 k_{2} \theta^{2}=-\frac{2 k_{2}}{\pi^{2} w^{4}}-\frac{h_{p} \varepsilon_{2} b^{2}}{\pi^{2} w^{2}}+\frac{32 h_{s} \varepsilon_{2} b^{4}}{45 \pi^{2} I_{s} w^{2}},
\end{gathered}
$$

The obtained Eq. (8) generalize the case discussed in paper [5] - here we consider a more general spatial profile and assume the propagation in a CQ medium.

Introducing the trial function (4) into the integral:

$$
P(z)=\int_{-\infty}^{\infty} \int_{-\infty}^{\infty} \int_{-\infty}^{\infty}|U|^{2} d x d y d t=2 \pi \int_{0}^{\infty} r d r \int_{-\infty}^{\infty} R^{2} d t
$$

We recognize the expression in Eq. (8a) as $P(z)$, so:

$$
2 \pi a^{2} b^{2} w=P=\text { const. }
$$

The following Eq. (8b) describes the changes of the propagation term. Since this term does not appear in the remaining equations, we will not take this equation into account further. Equations (8c) and (8e) express the spatial and temporal curvature by the speed of changes of the corresponding width. Using these expressions to eliminate curvatures from Eqs. (8d) and (8f), we derive $[6,7]:$

$$
\begin{gathered}
a^{\prime \prime}=-\Omega^{2} a+\frac{1}{k^{2} a^{3}}-\frac{h_{p} \varepsilon_{2} b^{2}}{3 h_{a} k a}+\frac{32 h_{s} \varepsilon_{2} b^{4}}{135 h_{a} k I_{s} a}, \\
w^{\prime \prime}=\frac{4 k_{2}^{2}}{\pi^{2} w^{3}}+\frac{2 h_{p} k_{2} \varepsilon_{2} b^{2}}{\pi^{2} w}-\frac{64 h_{s} k_{2} \varepsilon_{2} b^{4}}{45 \pi^{2} I_{s} w^{2}} .
\end{gathered}
$$

Let us analyze the stationary solution of the above system $b(z)=b_{0}, a(\mathrm{z})=a_{0}$ and $w(z)=w_{0}$. To simplify its form we introduce the normalized quantities: $A_{0}=\sqrt{k \Omega} \cdot a_{0}$,

$W_{0}=\sqrt{\Omega /\left|k_{2}\right|} \cdot w_{0}, B_{0}=\sqrt{\left|\varepsilon_{2}\right| /(2 \Omega)} \cdot b_{0}, \quad \Delta=64 \Omega /\left(45\left|\varepsilon_{2}\right| I_{s}\right)$ and $P=k\left|\varepsilon_{2}\right| \sqrt{\Omega /\left|k_{2}\right|} /(4 \pi) \cdot N-$ all of them positive and properly defined for both focusing and defocusing medium case. The solution of the system (10b)-(10c) expressed as a function of normalized width has the form:

$$
\begin{gathered}
B_{0}=\sqrt{\frac{h_{p}}{2 h_{s} \Delta}\left(1-\sqrt{1-\frac{4 h_{s} \Delta}{h_{p}^{2} W_{0}^{2}}}\right)}, \\
A_{0}=\sqrt{\sqrt{1+\frac{1}{9 h_{a}^{2} W_{0}^{4}}}-\frac{\operatorname{sgn}\left(\varepsilon_{2}\right)}{3 h_{a} W_{0}^{2}}}, \\
N=B_{0}^{2} A_{0}^{2} W_{0} .
\end{gathered}
$$

In Fig. 1 we plot the obtained solutions as functions of the stationary pulse height $B_{0}$ for the case of $\operatorname{Kerr}(\Delta=0)$ and CQ focusing medium with $\Delta=0.2$. Only for small fields the behaviour of functions in these two cases is similar - for large fields both widths diminish in the Kerr case and increase up to infinity in the CQ case (for a defocusing medium and increasing field $A_{0} \rightarrow \infty$ in the Kerr case and $A_{0} \rightarrow 0$ in a CQ medium). As the boundary between small and large fields we can take $b_{q}=\sqrt{I_{s} / 2}$ (black lines in figures) - for this value nonlinear CQ permittivity obtains its maximum. Observe that the spatial width $A_{0}$ depends on saturation parameter $\Delta$ and very weakly on field for higher order pulses. Moreover, we can see that stationary pulses with a sufficiently strong field cannot propagate in CQ media - their temporal width becomes infinite for a finite field (dotted blue and green lines in figures).

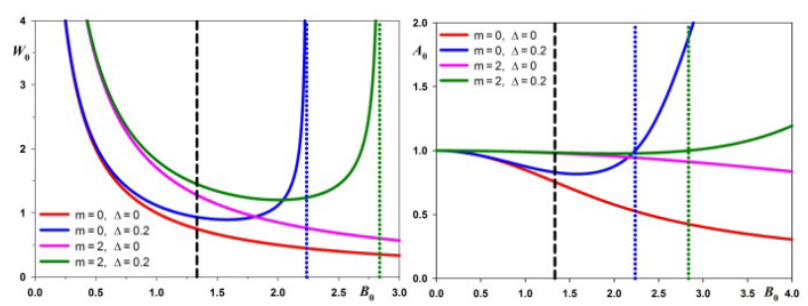

Fig. 1. Stationary temporal and spatial width of 0 -th and 2 order pulse. Dashed lines separate regimes of small and large fields, dotted lines indicate maximum fields (CQ case).

Equations (10b)-(10c) for the non-stationary case cannot be solved analytically, but we are able to solve them assuming small deviations from the non-stationary case:

$$
\begin{aligned}
a(z)=a_{0}+\delta a(z), & & |\delta a(z)| & <<a_{0}, \\
w(z)=w_{0}+\delta w(z), & & |\delta w(z)| & <<w_{0} .
\end{aligned}
$$

Linearization of the system of Eqs. (10b)-(10c) gives:

$$
\left[\begin{array}{l}
\delta a^{\prime \prime} \\
\delta w^{\prime \prime}
\end{array}\right]=\Omega^{2} \cdot\left[\begin{array}{ll}
\mathrm{M}_{a a} & \mathrm{M}_{a w} \\
\mathrm{M}_{w a} & \mathrm{M}_{w w}
\end{array}\right]\left[\begin{array}{l}
\delta a \\
\delta w
\end{array}\right]
$$

with coefficients $\mathrm{M}_{i j}(i, j=a, w)$ expressed by:

$$
\begin{gathered}
\mathrm{M}_{a a}=-4-\frac{16 h_{s} \varepsilon_{2} P^{2}}{135 \pi^{2} h_{a} k \Omega^{2} I_{s} w_{0}^{2} a_{0}^{6}} \\
\mathrm{M}_{w a}=\frac{16 k_{2}^{2}}{\pi^{2} \Omega^{2} a_{0} w_{0}^{3}}\left(1+\frac{h_{p} \varepsilon_{2} P w_{0}}{8 \pi k_{2} a_{0}^{2}}\right)=-\frac{12 h_{a} k k_{2}}{\pi^{2}} \mathrm{M}_{a w} \\
\mathrm{M}_{w w}=\frac{h_{p} k_{2} \varepsilon_{2} P}{\pi^{3} \Omega^{2} a_{0}^{2} w_{0}^{3}} .
\end{gathered}
$$

The system (13) possesses the solution of an oscillatory or exponential type - it depends on the signs and relation between $\mathrm{M}_{i j}$. Let us consider small oscillations near stationary solutions:

$$
\left[\begin{array}{l}
\delta a(z) \\
\delta w(z)
\end{array}\right]=\left[\begin{array}{l}
\delta a(0) \\
\delta w(0)
\end{array}\right] \cdot e^{i \Omega f z}
$$


where $f$ denotes normalized frequency. After introducing (15) into (13), we obtain the quotient of initial amplitudes:

$$
\frac{\delta a(0)}{\delta w(0)}=-\frac{\mathrm{M}_{w w}+f^{2}}{\mathrm{M}_{w a}}
$$

and two solutions for frequency corresponding to the same pulse parameters:

$$
\begin{aligned}
& f_{1}^{2}=-\frac{\mathrm{M}_{a a}+\mathrm{M}_{w w}}{2}+\sqrt{\left(\frac{\mathrm{M}_{a a}-\mathrm{M}_{w w}}{2}\right)^{2}+\mathrm{M}_{a w} \mathrm{M}_{w a}}, \\
& f_{2}^{2}=-\frac{\mathrm{M}_{a a}+\mathrm{M}_{w w}}{2}-\sqrt{\left(\frac{\mathrm{M}_{a a}-\mathrm{M}_{w w}}{2}\right)^{2}+\mathrm{M}_{a w} \mathrm{M}_{w a}} .
\end{aligned}
$$

Any of two solutions (16) determine normal oscillations - analogous to that known from mechanics. Let us introduce $\mathrm{M}_{i j}$ given by (14) into Eqs. (16) and replace $P$, $w_{0}, a_{0}$ and $I_{s}$ by normalized quantities $N, W_{0}, A_{0}$ and $\Delta$. In spite of the fact that the resulting formulas are cumbersome, they enable to inspect the behaviour of small oscillations directly as functions of field height $B_{0}$. Figures 2 and 3 show the obtained frequencies as functions of pulse height in a focusing and defocusing medium. Observe that for small fields the oscillations in both types of media are very similar - those of small frequency vanish with a vanishing field while the others practically remain constant in a wide field regime, receiving the value $f \cong 2$.

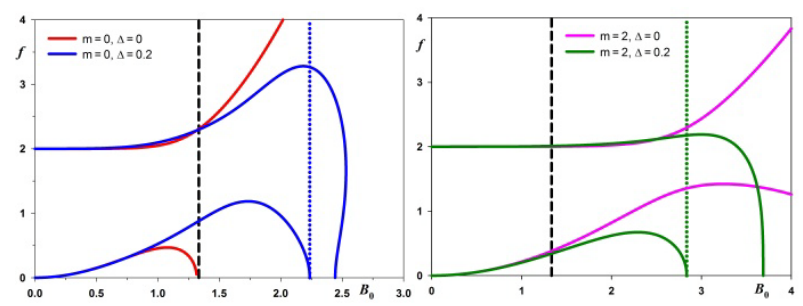

Fig. 2. Frequencies of normal oscillations of 0 -th and 2 order pulse in focusing medium.

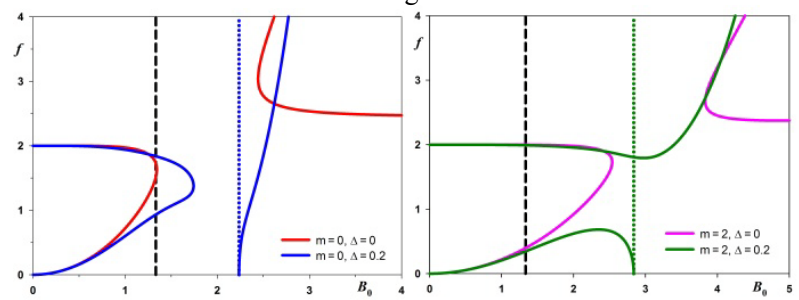

Fig. 3. Frequencies of normal oscillations of 0 -th and 2 order pulse in defocusing medium.

For larger fields, the oscillations differ. In a defocusing medium we obtain two regimes of oscillations divided by a gap between them (in the gap Eq. (13) has exponential solutions). However, for larger saturation and larger pulse orders two regimes can join. In a focusing medium saturation stabilizes oscillations - they exist in the whole regime of permissible stationary parameters, while in the Kerr medium for a sufficiently strong field the solution of Eq. (13) has a mixed oscillatory-exponential character.
Generally, the evolution of any pulse parameter is described by a superposition of normal oscillations with amplitude depending on the initial condition. In the left part of Fig. 4 we plot numerical solutions $A(z)$ and $W(z)$ of Eqs.(10) without any approximation. The superposition of two oscillations with different frequencies gives beating, which is easily observed. Therefore the existence of two normal oscillations is an immanent feature of Eqs. (10) and does not appear because of the approximation. The right plot of Fig. (4) shows one more solution of Eqs. (10) - irregular oscillations exist during the first few periods and then their temporal width infinitely increases and the pulse is transformed into a regularly oscillating $(2+1) \mathrm{D}$ spatial beam propagating in a graded-index waveguide.

Equations (13) and (10) also possess solutions in which both widths diminish exponentially, which results in pulse collapse. We observe such collapse in a Kerr focusing medium for initial parameters away from stationary ones.
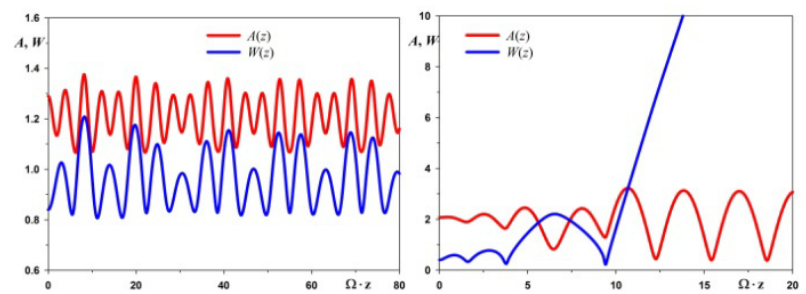

Fig. 4. Superposition of normal oscillations of the 0 -th order pulse in a defocusing medium - a stable and unstable case.

To summarize, the canonical method applied to $(3+1) \mathrm{D}$ pulses with axial symmetry propagating in a CQ medium with a linear graded-index profile enables us to analyze the properties of stationary and non-stationary propagation very effectively. An adequate profile of linear permittivity supports pulse oscillations in both focusing and defocusing medium for quite a large range of pulse parameters. An analytical description of evolution is possible in small oscillations approximation and predicts a few scenarios of pulse behavior - regular oscillations, infinite growth of temporal width and even pulse collapse. The quantitative determination of regimes of such scenarios is not very precise in this approximation, but a qualitative description agrees quite well.

\section{References}

[1] G.P. Agrawal, Nonlinear Fiber Optics, (Academic Press, San Diego 2001).

[2] V. Skarka, V.I. Berezhiani, R. Miklaszewski, Phys. Rev. E. 56, 1080 (1997)

[3] D. E. Edmundson, R. H. Enns, Phys. Rev. A 51, 2491 (1995).

[4] D. Mihalache, D. Mazilu, L.-C. Crasovan, B.A. Malomed, F. Lederer, Phys. Rev. E 61, 7142 (2000).

[5] S. Raghavan, G.P. Agraval, Opt. Commun. 180, 377 (2000).

[6] J. Jasinski, Opto-Electron. Rev. 13, 129 (2005).

[7] J. Jasiński, M. Kościesza, Proc. SPIE 7141, 7141G (2008).

[8] G.A. Korn, T.M. Korn, Mathematical Handbook for scientists and engineers, (McGraw-Hill, New York 1968). 